\title{
Targeting lentiviral vector to specific cell types through surface displayed single chain antibody and fusogenic molecule
}

\author{
Yuning Lei, Kye-II Joo, Jonathan Zarzar, Clement Wong, Pin Wang*
}

\begin{abstract}
Background: Viral delivery remains one of the most commonly used techniques today in the field of gene therapy. However, one of the remaining hurdles is the off-targeting effect of viral delivery. To overcome this obstacle, we recently developed a method to incorporate an antibody and a fusogenic molecule (FM) as two distinct molecules into the lentiviral surface. In this report, we expand this strategy to utilize a single chain antibody (SCAb) for targeted transduction.

Results: Two versions of the SCAb were generated to pair with our various engineered FMs by linking the heavy chain and the light chain variable domains of the anti-CD20 antibody ( $\alpha$ CD20) via a GS linker and fusing them to the hinge- $\mathrm{CH} 2-\mathrm{CH} 3$ region of human $\mathrm{IgG}$. The resulting protein was fused to either a HLA-A2 transmembrane domain or a VSVG transmembrane domain for anchoring purpose. Lentiviral vectors generated with either version of the SCAb and a selected FM were then characterized for binding and fusion activities in CD20-expressing cells.

Conclusion: Certain combinations of the SCAb with various FMs could result in an increase in viral transduction. This two-molecule lentiviral vector system design allows for parallel optimization of the SCAb and FMs to improve targeted gene delivery.
\end{abstract}

\section{Introduction}

Gene therapy is the introduction of a functional gene into a dysfunctional cell for a therapeutic benefit. To date, viral vectors remain the most commonly used gene delivery vehicles due to their high transduction efficiencies $[1,2]$. In particular, lentiviral vectors represent one of the most effective gene delivery vehicles as they allow for stable long-term transgene expression in both dividing and non-dividing cells. In order to expand the targeted specificity of viral vectors beyond their natural tropism, numerous studies have been focused on pseudotyping lentiviral vectors with envelope glycoproteins derived from other viruses, such as the glycoprotein from vesicular stomatitis virus (VSVG) [3,4]. However, since the VSVG is thought to recognize a ubiquitous membrane phospholipids instead of a unique cellular receptor, pseudotyping generates vectors with broad specificities $[5,6]$. To mitigate this off-target effect,

\footnotetext{
* Correspondence: pinwang@usc.edu

Mork Family Department of Chemical Engineering and Materials Science, University of Southern California, Los Angeles, CA 90089, USA
}

previous attempts have been devoted to engineer the viral glycoprotein to recognize a specific cellular target by insertion of ligands, peptides, or antibodies [7-16]. Another approach involves bridging the viruses and the targeted cell with ligand proteins or antibodies [17-20]. However, these modifications to the surface glycoprotein appear to perturb the natural fusion function of the glycoprotein, resulting in a reduction of transduction efficiency.

Recently, our lab has developed a strategy to target lentiviral vectors to specific cell types by incorporating a surface antibody specific to CD20 antigen and a fusogenic molecule (FM) as two distinct molecules [21]. Kielian and co-workers reported several versions of the Sindbis virus glycoprotein that were less dependent on cholesterol for transduction [22]. We applied these mutations (E1 226) to the binding defective Sindbis glycoprotein and observed that they were able to enhance transduction efficiency when paired with an anti-CD20 antibody ( $\alpha$ CD20) [23]. In this study, we report our attempt to utilize a single chain antibody (SCAb) to pair 
with a FM for targeting lentiviral vectors. Our SCAb is composed of variable domains of the heavy and light chains of $\alpha \mathrm{CD} 20$, linked by a GS linker and fused to a hinge- $\mathrm{CH} 2-\mathrm{CH} 3$ region of human IgG. To anchor the $\mathrm{SCAb}$ onto the viral surface, we conjugated the SCAb with either the HLA-A2 transmembrane domain (SC2H7-A2) or the VSVG transmembrane domain (SC2H7-GS). We demonstrated that the lentiviral vector enveloped with either of these antibody configurations could achieve targeted transduction to CD20-expressing cells. We also compared the targeted transduction efficiency and the binding avidity of both versions of the $\mathrm{SCAb}$ and investigate the molecular roles of the displayed proteins in mediating lentiviral transduction.

\section{Results}

\section{Construction of SCAb for targeting}

We have previously demonstrated that targeting lentiviral vectors can be generated by co-transfecting producer cells with a lentiviral vector backbone plasmid, FUGW, a plasmid encoding an antibody's heavy and light chains, a plasmid encoding antibody accessory proteins, and a plasmid encoding a FM, along with lentiviral packaging plasmids $[21,24]$. In this report, we wanted to expand the targeting strategy by pairing FMs with SCAbs. To generate the SCAb for this study, we first PCR-amplified the light chain and heavy chain variable regions of the $\alpha \mathrm{CD} 20$ and linked them with a GS linker. To allow for the formation of disulfide-linked dimmers to stabilize the SCAb, the hinge- $\mathrm{CH} 2-\mathrm{CH} 3$ region of the human IgG was fused to the heavy chain variable region [25-28]. To anchor the SCAb, the HLAA2 transmembrane domain or the VSVG transmembrane domain was added to the $\mathrm{C}$-terminal and the resulting constructs were designated as SC2H7-A2 and SC2H7-GS, respectively (Fig. 1).

\section{Production of lentiviral vectors}

We generated SCAb-bearing lentiviral vectors (FUGW/ SC2H7-A2/FM or FUGW/SC2H7-GS/FM) by co-transfecting $293 \mathrm{~T}$ cells with the lentiviral backbone plasmid FUGW, a FM-encoding plasmid (SINmu, SGN, SGM, or AGM), and a plasmid encoding the described SCAb (pSC2H7-A2, or pSC2H7-GS) along with other necessary packaging plasmids (Fig. 1). Independently, a lentiviral vector bearing an isotype control antibody, pAB and a FM was produced as a non-target control. Furthermore, we included a VSVG-pseudotyped lentiviral vector, FUGW/VSVG as an additional positive control since VSVG-carrying viral vectors are known to transduce a variety of different cell types [4]. As shown in Fig. 2A, FACS analysis of transfected, virus-producing $293 \mathrm{~T}$ cells showed that virtually all of the cells were able to be transfected with the viral backbone plasmid
FUGW. Among the GFP-positive cells, roughly 25\% to $40 \%$ of the producer cells were positive for both the antibody and the FM (Fig. 2B). As expected, transfection with VSVG as the envelope protein showed no expression of the FM and the SCAb. The similar levels of transfection and expression of the four FMs suggests that they could be incorporated into the lentiviral surface with similar efficiency.

\section{Incorporation of SCAb and FM onto lentiviral vectors}

A virus-cell binding assay was performed to evaluate SCAb-mediated binding to CD20-expressing cells. As a target, we used a $293 \mathrm{~T}$ cell line stably expressing the CD20 antigen (designated as 293T/CD20). The parental cell line 293T served as a negative control. The lentiviral vector, FUW/SC2H7-A2/SGN or FUW/VSVG, was incubated with either the target cell line, 293T/CD20, or the control cell line, 293T, for one hour at $4{ }^{\circ} \mathrm{C}$, after which, the cell-virus complex was fixed with $4 \%$ formaldehyde and stained by an anti-p24 antibody to detect the viral core and 4',6-diamidino-2-phenylindole (DAPI) for nucleus. As shown in Fig. 3A, confocal images revealed that the lentiviral vector (FUW/SC2H7-A2/SGN) was able to bind to $293 \mathrm{~T} / \mathrm{CD} 20$ cell line, but not to the control 293T cell line. In contrast, the lentiviral vector FUW/VSVG was able to bind to both 293T and 293T/ CD20 cell lines. In addition, a quantitative virus-cell binding assay was conducted to evaluate SCAb-mediated binding to CD20-expressing cells. Lentiviral vectors (FUGW/SC2H7-A2/FM, FUGW/SC2H7-GS/FM, and FUGW/AB/FM) were incubated with either 293T or $293 \mathrm{~T} / \mathrm{CD} 20$ cells for one hour at $4{ }^{\circ} \mathrm{C}$ to prevent internalization of the viral particle. Cells were then stained for the presence of viral particles on the cell surface using an anti-FM antibody and quantified using flow cytometry. As shown in Fig. 3B, flow cytometry analysis showed that the vector of either FUGW/SC2H7-A2/FM or FUGW/ SC2H7-GS/FM was able to bind to $293 \mathrm{~T} / \mathrm{CD} 20$ cells. FACS analysis also showed that the virus bound to the 293T/CD20 cell surface displayed the FMs (Fig. 3B), suggesting that both SCAb and FM were incorporated on the same virion. Additionally, the control 293T cells showed no detectable FM, confirming that the observed viral particle binding to the cells is indeed due to the SCAb-antigen interaction. Similarly, a non-targeting lentiviral vector FUGW/AB/FM was unable to bind to either $293 \mathrm{~T}$ or $293 \mathrm{~T} / \mathrm{CD} 20$ cells.

\section{Targeted transduction of lentiviral vectors}

We conducted transduction experiments to evaluate the efficiency of lentiviral vectors bearing both SCAb and FM to transduce the CD20-expressing cell line. The lentiviral vector bearing VSVG was used as a positive control, whereas the lentiviral vector co-displaying $A B$ and 


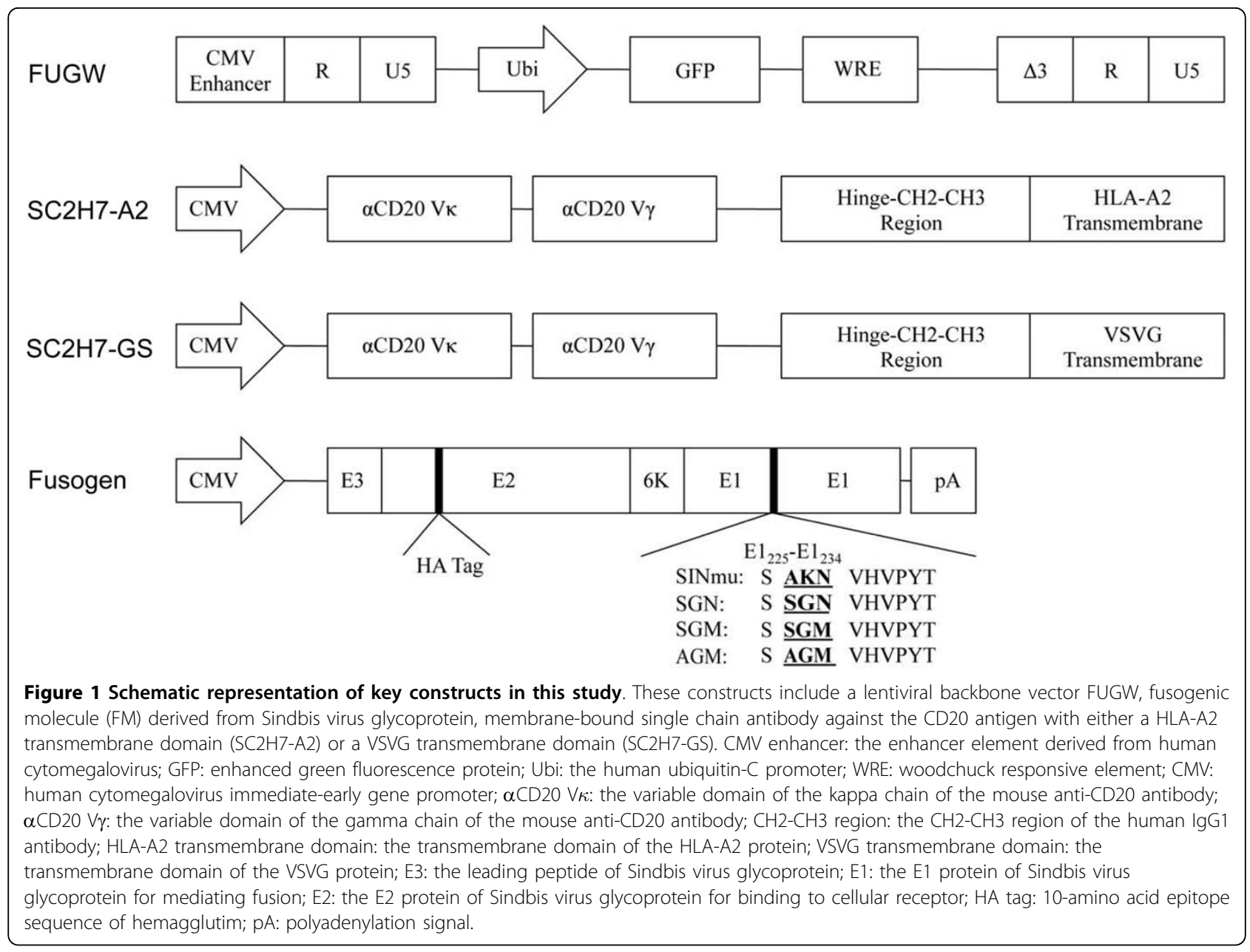

FM was included as a negative control. Cell lines were transduced by indicated lentiviral vectors and analyzed by FACS five days post-transduction. The GFP expression level was detected to quantify the specificity and efficiency.

Recombinant lentiviral vectors bearing both SCAb and FM were able to specifically transduce the $293 \mathrm{~T} / \mathrm{CD} 20$ cell line with various efficiencies $(15 \% \sim 30 \%)$ varying upon the choice of the FMs (Fig. 4A). In contrast, less than $5 \%$ of transduction efficiency was observed for the $293 \mathrm{~T}$ cell line. In addition, the titer of FUGW/SC2H7A2/SGN was estimated to be $\sim 0.15 \times 10^{6}$ transduction units $(\mathrm{TU}) / \mathrm{mL}$ on the $293 \mathrm{~T} / \mathrm{CD} 20$ cells (Fig. 4B); the titer was determined in the dilution ranges that showed a linear response of GFP expression with viral serial dilution. In another control experiment, when the lentiviral vector bearing an isotype antibody paired with a FM (FUGW/AB/FM) were used, less than $5 \%$ of cells were transduced to express GFP. This finding further highlighted the significance of antibody-directed transduction. No transduction was observed with the lentiviral vector containing only SCAb, indicating the necessity of FM to complete transduction. Thus, lentiviral vectors must display both SCAb and FM for efficient transduction to target cells.

Among the various lentiviral vectors bearing the same SCAb but different FMs, different transduction efficiencies were observed. The lentiviral vector displaying SC2H7-A2 and SINmu exhibited 15\% transduction efficiency. However, lentiviral vectors displaying other FMs (SGN, SGM, and AGM) resulted in specific transductions of $25 \%$ to $30 \%$. A similar trend was observed in another independent study where the SCAb with VSVG transmembrane domain was used as the targeting antibody (SC2H7-GS) (Fig. 4A). In this case, the lentiviral vector bearing SINmu and SC2H7-GS was able to specifically transduce about $14 \%$ of the $293 \mathrm{~T} / \mathrm{CD} 20$ cells, whereas the specific transduction efficiency was increased to 25\% when other FMs (SGN, SGM and AGM) were used in combination with the SC2H7-GS.

\section{Assays for studying the entry mechanism}

We hypothesized that our engineered lentiviral vector entered cells via receptor-mediated endocytosis followed 


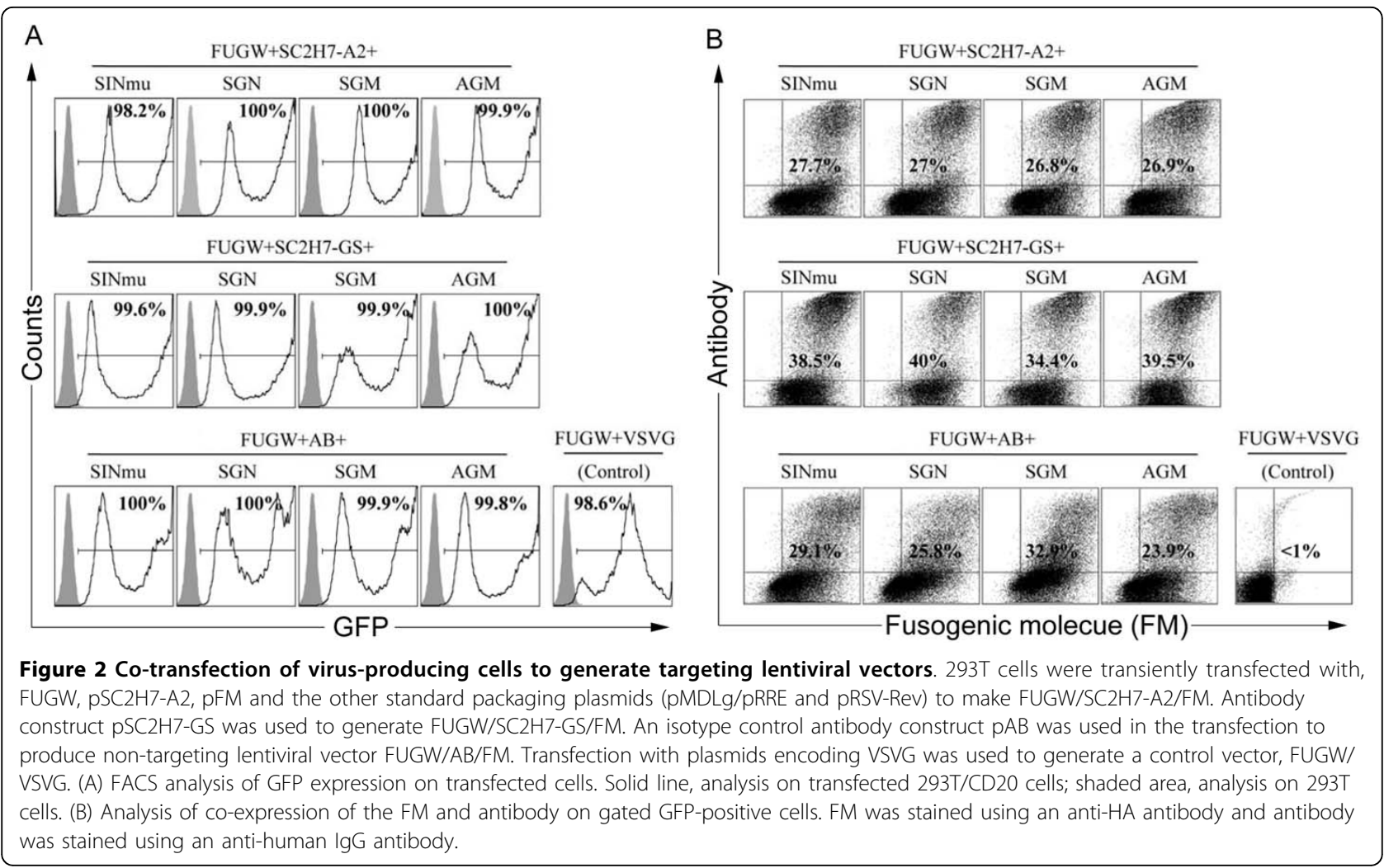

by the endosomal fusion leading to the release of the vector core. To validate our hypothesis, we designed two independent experiments to study these two critical steps. Since the combination of SGN and SC2H7-A2 showed the greatest targeting efficiency in the transduction experiment, we chose this combination for the study of the entry mechanism. 293T/CD20 cells were exposed to either FUGW/SC2H7-A2/SGN or FUGW/ VSVG in the presence of various amount of either soluble $\alpha \mathrm{CD} 20$ or isotype control antibody (Fig. 5B). As expected, the level of transduction efficiency (FUGW/ SC2H7-A2/SGN) dropped as the concentration of the soluble $\alpha \mathrm{CD} 20$ increased, whereas no noticeable reduction in transduction efficiency was observed when the isotype control was used. In contrast, transduction efficiency of FUGW/VSVG was not affected by soluble $\alpha \mathrm{CD} 20$.

The second critical step of transduction pathway involved the $\mathrm{pH}$-dependent fusion event leading to the release of the viral core. To verify the $\mathrm{pH}$ requirement, we incubated either FUGW/SC2H7-A2/SGN or FUGW/ GP160 with 293T/CD20 or Ghost-CCR5 cells in the increased presence of bafilomycin, which can raise the $\mathrm{pH}$ of the endosomal compartment. We observed a dramatic decrease in transduction efficiency (FUGW/ SC2H7-A2/SGN) in response to increasing amount of bafilomycin (Fig. 5A). In a control experiment where a
pH-independent virus (FUGW/GP160) was used, an increase in transduction efficiency was observed, which was consistent with previously published data $[29,30]$. Thus, the $\mathrm{pH}$ in the endosomal compartment is critical for viral membrane fusion.

\section{pH dependency study on the FMs}

As shown from the targeted transduction experiment, lentiviral vectors enveloped with various FMs resulted in different targeting efficiency (Fig. 4). We thus designed a liposome-virus fusion experiment to characterize the fusion property of these FMs. As shown in Fig. 6, roughly $40 \%$ to $50 \%$ of the lentiviral vector (FUGW/ $\mathrm{SC} 2 \mathrm{H} 7-\mathrm{A} 2 / \mathrm{FM}$ ) fused at $\mathrm{pH}$ of 5.6. When the same experiment was performed at $\mathrm{pH}$ environment of 6.2, only $12 \%$ of the lentiviral vector bearing SINmu fused, whereas a $40 \%$ to $50 \%$ fusion activity was obtained for vectors bearing other FMs (SGN, SGM, and AGM). Correlating the liposome-virus experiment with the targeted transduction experiment (Fig. 4), we observe a clear trend showing that the higher fusion activity of the FMs results in a higher transduction efficiency.

\section{Binding avidity of lentiviral vectors to target cells}

In order to understand the different transduction efficiency of lentiviral vectors bearing these two different versions of SCAb (SC2H7-A2 and SC2H7-GS), we 


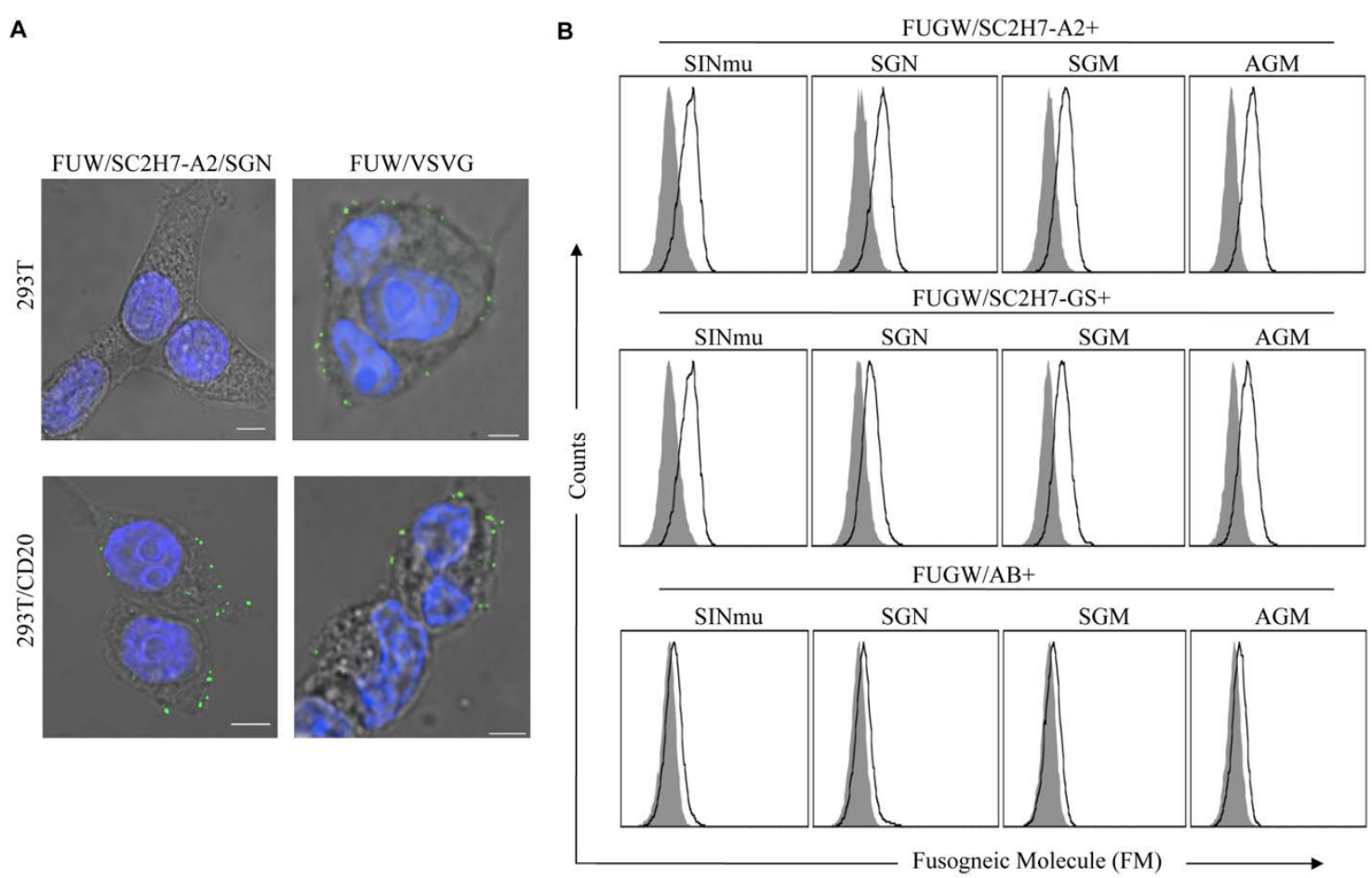

Figure 3 Incorporation of both FM and antibody onto the vector surface. (A) 293T (top) and 293T/CD20 (bottom) cells were incubated with either FUGW/SC2H7-A2/SGN (left) or FUGWNSVG (right) at $4^{\circ} \mathrm{C}$ for 1 hour, fixed and immunostained with anti-p24 antibody (green) and DAPI nuclear staining (blue). Images were acquired with a laser scanning confocal microscope. Scale bar represents $2 \mu$ m. (B) Co-expression of antibody and FM on the same viral surface. 293T (shaded area) or 293T/CD20 (solid line) cells were incubated with FUGW/SC2H7-A2/FM, FUGW/ $\mathrm{SC} 2 \mathrm{H} 7-\mathrm{GS} / \mathrm{FM}$ or FUGW/AB/FM at $4^{\circ} \mathrm{C}$ for 1 hour, followed by staining of FM by anti-HA antibody. The binding of the virus to the cells was detected by FACS analysis.

conducted a binding avidity assay. Increasing amount of the lentiviral vectors (FUGW/SC2H7-A2/SGN and FUGW/SC2H7-GS/SGN) were incubated with 293T/ CD20 cells followed by the surface staining of the FM. The geometry mean fluorescence (GMF) intensity was measured and scatchard analysis was performed to determine the avidity of the lentiviral vector to bind to 293T/CD20 cells (Fig. 7A). In agreement with our transduction experiment (Fig. 4), the SC2H7-A2-enveloped lentiviral vector showed slightly better binding avidity to the target cells as compared to that of the SC2H7-GSenveloped lentiviral vector. We also noted that when SC2H7-A2 was used to envelope the lentiviral vector, the vector production was increased as compared to that of SC2H7-GS (Fig. 7B). These findings explain the result of transduction experiment where the lentiviral vector pseudotyped with the SC2H7-A2 antibody showed higher transduction efficiency as compared to that of SC2H7-GS-bearing vector (Fig. 4).

\section{Discussion}

The purpose of this study is to incorporate both membrane-bound SCAb and FM on the lentiviral surface to achieve targeted transduction to specific cell types.
Previously, we reported a strategy of separating the binding and fusion functions of viral glycoprotein for cell specific targeting [21]. By pairing the $\alpha$ CD20 with a more fusion active FM, the resulted lentiviral vectors showed enhanced transduction [23]. In this study, we extended the targeting strategy to utilize a membranebound SCAb with the engineered FMs. Insertion of $\mathrm{SCAb}$ into the viral glycoprotein has shown to be able to redirect vector particles to specific cellular target $[8,9,13]$. However, these modifications usually resulted in reduced transduction efficiency. Our strategy of separating binding and fusion functions allows us to engineer a targeting lentiviral vector system by optimizing these two parameters in parallel without compromising their functions.

The lentiviral vectors bearing both SCAb and FM can specifically transduce CD20-expressing cells. The specific transduction occurs through a two-step process. First the virus must recognize and bind to CD20-expressing cells. Using flow cytometry and confocal microscopy, we verified that the SCAb was able to mediate the binding of the vector to the CD20 antigen on the cellular surface. Furthermore, the soluble $\alpha \mathrm{CD} 20$ inhibition assay revealed that the targeting kinetics of the $\mathrm{SCAb}$ vector 


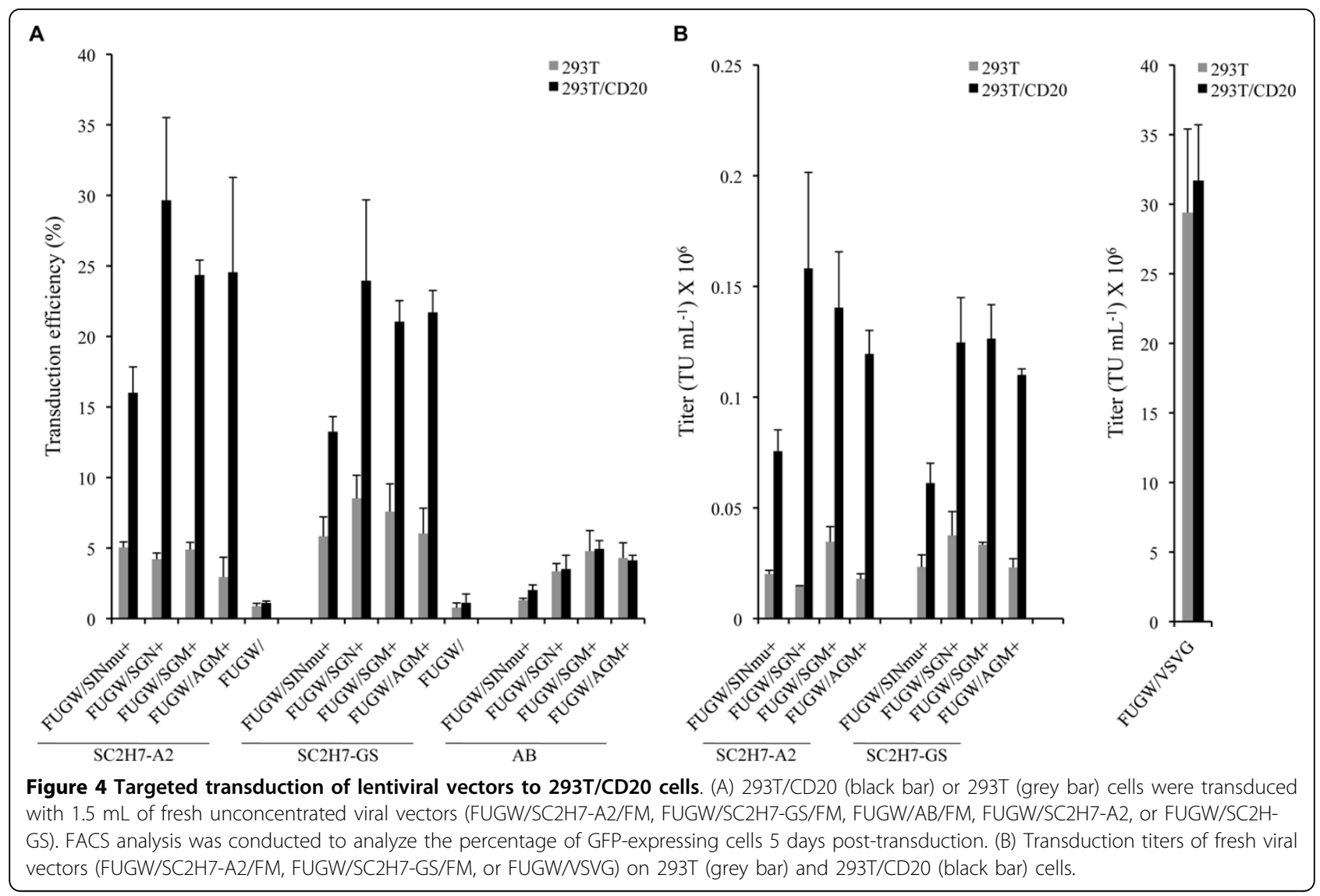

was inhibited in a dose-dependent fashion, confirming the binding requirement for the observed targeting. The second step for targeted transduction is the FMmediated endosomal fusion to deliver the viral payload into the cell. A high titer and efficient transduction demonstrated that the FM was functional when combined with SCAb on the viral surface. Thus, the targeting lentiviral vector succeeds in these two steps to achieve efficient transduction.

As suggested from our previous studies, two different approaches can be applied to further optimize this twomolecule targeting strategy. By engineering the fusion loop of the SINmu, transduction can be enhanced [23]. Lentiviral vectors incorporating SCAb and SINmu consistently yielded lower transduction efficiency as compared to viral vectors with other FMs (SGN, SGM, or AGM). The difference in transduction efficiency may have resulted from the endosomal fusion kinetics of the different FMs. Recent studies of alphavirus glycoproteins have indicated that mutation in the E1 fusion domain might favor an increase in endosomal fusion ability [31-33]. We suspected that our mutation in the E1 domain might have a similar role in lowering the activation energy for the fusion event. Our liposome-virus fusion assay revealed that SINmu was not fusion-active at $\mathrm{pH}=6.2$, while other FMs were active at this $\mathrm{pH}$. This direct correlation between the $\mathrm{pH}$ of fusion and the transduction efficiency suggests that the FMs that are more active at a higher $\mathrm{pH}$ can have better capacity to mediate lentiviral transduction. Consequently, targeted transduction may be further improved by constructing a library of FMs and screening for a FM with higher $\mathrm{pH}$ fusion activity.

Another approach to optimize this two-molecule targeting strategy is to engineer the targeting antibody to be more efficiently incorporated onto the lentiviral vector surface. Having the targeting molecule more efficiently incorporated onto the vector surface could enhance the binding of the vector to the cognate receptor on the target cell surface, thereby increasing transduction efficiency. To enhance the display of SCAb onto the viral surface, we constructed two SCAbs, each fused to a different transmembrane domain: the HLA-A2 transmembrane domain or the VSVG transmembrane domain. The targeted transduction efficiency was consistently higher with the $\mathrm{SC} 2 \mathrm{H} 7-\mathrm{A} 2$-bearing vector. The binding avidity from the scatchard analysis revealed that the FUGW/SC2H7-A2/ SGN vector exhibited a slightly higher binding avidity as compared to FUGW/SC2H7-GS/SGN. The higher avidity of the $\mathrm{SC} 2 \mathrm{H} 7$-A2-bearing vector may be due to more 


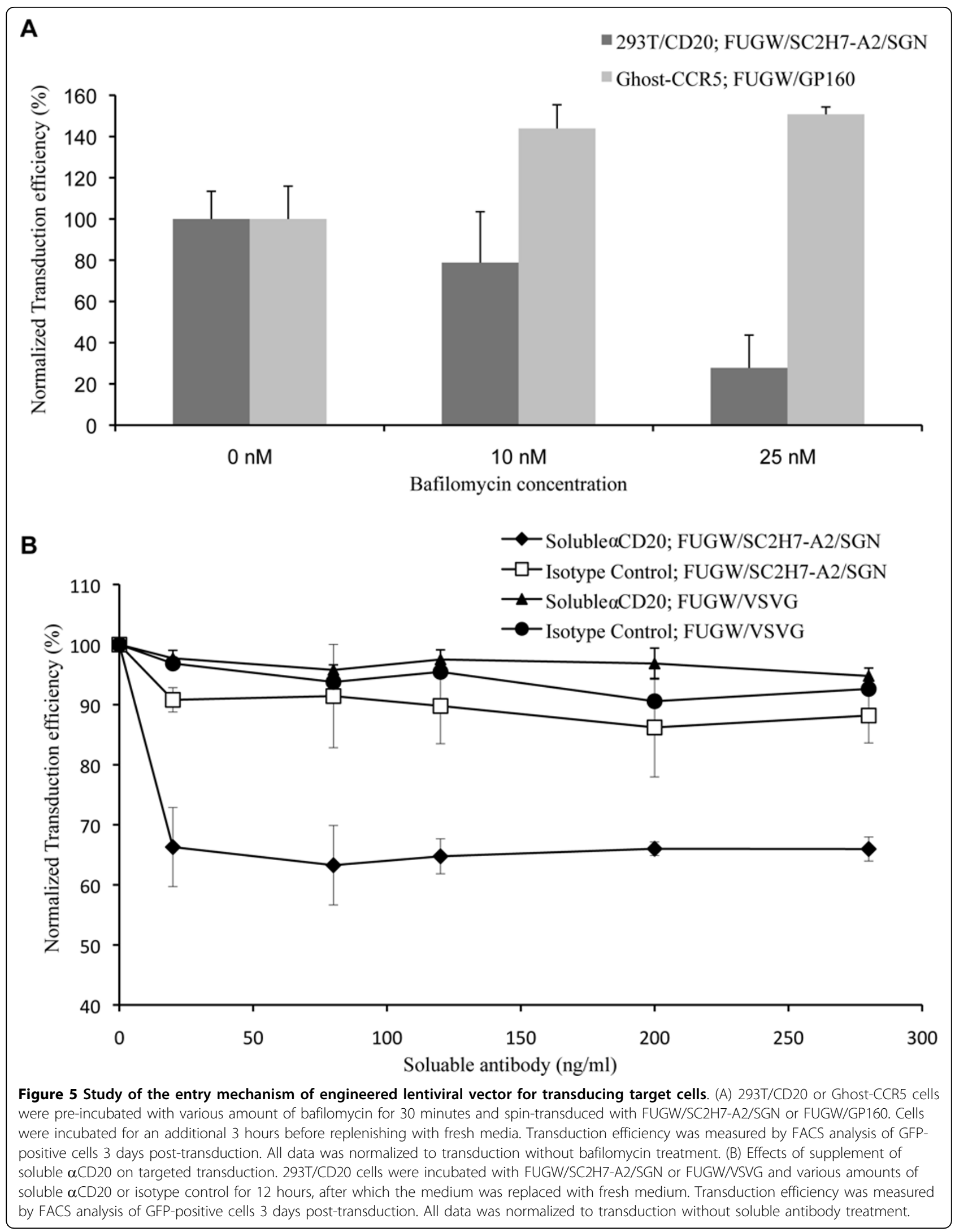




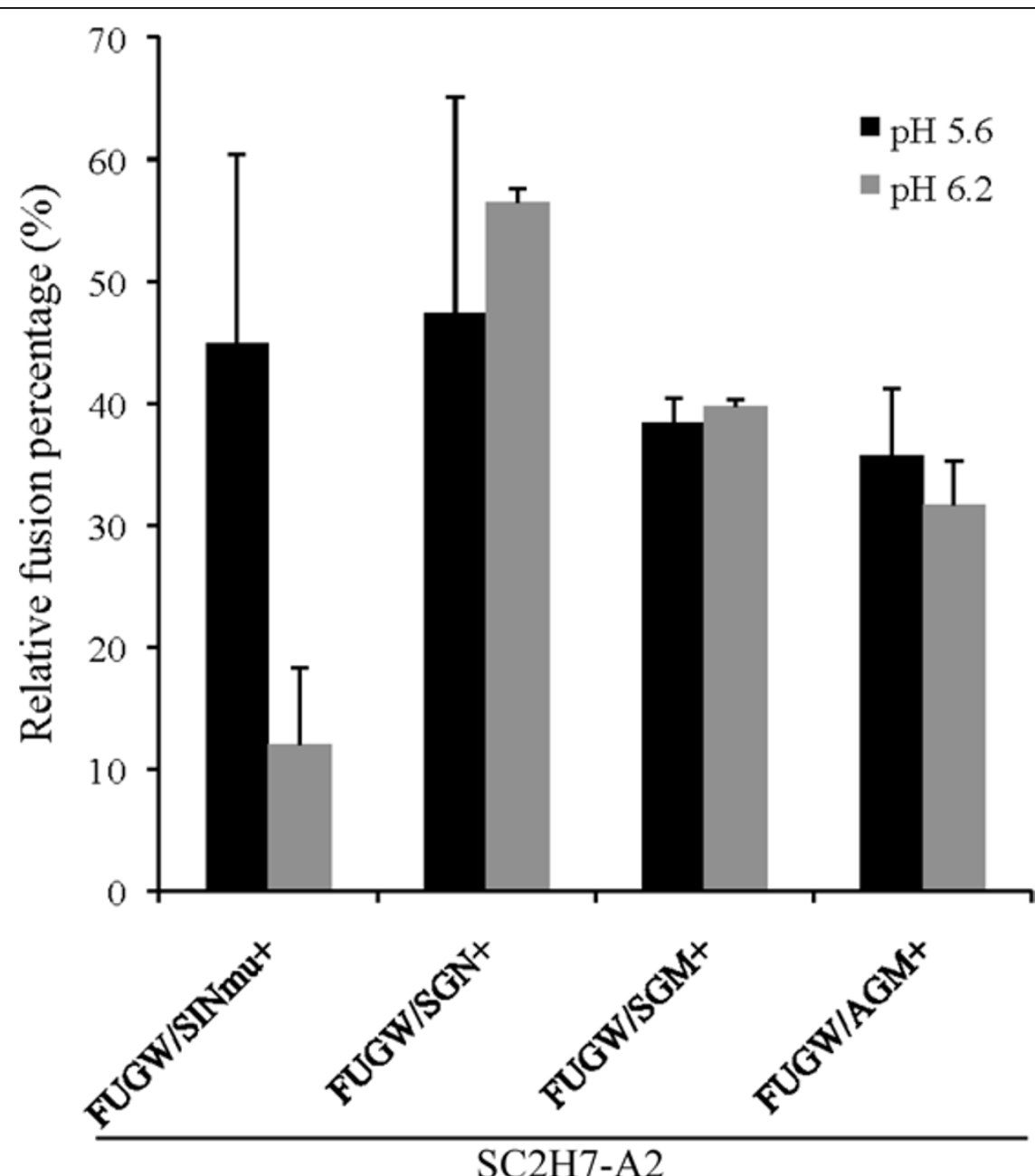

Figure $6 \mathrm{pH}$-dependent study of the fusion activity of various FMs. R18-labeled lentiviral vectors (FUGW/SC2H7-A2/FM) were mixed with liposomes $(200 \mu \mathrm{M})$ for 1 minute. Virus-liposome fusion was triggered by adding the appropriate volume of acetic acid and measured by dequenching of fluorescent R18 using a spectrofluorometer.

efficient incorporation of SC2H7-A2 onto the lentiviral vector surface. It has been proposed that lipid rafts can serve as assembly sites for the pseudotyped lentiviral vectors [34]. Recent studies have demonstrated a correlation between transmembrane domain and raft association with efficient viral incorporation [35]. Although these data indicate a role of the transmembrane interaction to facilitate more efficient incorporation onto the virus, further understanding is needed to identify the precise mechanism of the transmembrane to facilitate incorporation of both the SCAb and FMs.

\section{Materials and methods Construct preparation}

To generate the SCAb against the CD20 antigen, we first PCR-amplified the light chain variable region from an $\alpha \mathrm{CD} 20$ hybridoma cell line (ATCC, Manassas, VA, HB-9803) with primers CD20Lvfw (5'-CTG ACC CAG
ACC TGG GCG CAA ATT GTT CTC TCC CAG TCT CCA GCA ATC CTG TC-3') and CD20LvGSbw (5'CAC CTC CTG AAC CAC CGC CGC TAC CGC CTC CGC CTT TCA GCT CCA GCT TGG TCC CAG CAC C-3'). The HLA-A2 leading peptide sequence was then added to the 5 -end of the light chain variable region with primers HLA-A2 (5'-GAA CAA TTT GCG CCC AGG TCT GGG TCA GGG CCA GAG CCC CCG AGA GTA GCA GGA CGA GGG TTC-3') and HLAA2fw (5'-CTT AAG CTT ATG GCC GTC ATG GCG CCC CGA ACC CTC GTC CTG CTA CTC TCG GGG G-3'). We also PCR-amplified the heavy chain variable region with primers CD20hvGSfw (5'-GGT AGC GGC GGT GGT TCA GGA GGT GGC GGC AGT GGT GGA GGA TCT CAG GCT TAT CTA CAG CAG TCT GGG GCT GAG CTG-3') and CD20hvbw (5'-GTT TTG TCA CAA GAT TTG GGC TCA ACT GAA GAG ACG GTG ACC GTG GTC CCT GTG-3'). The PCR 


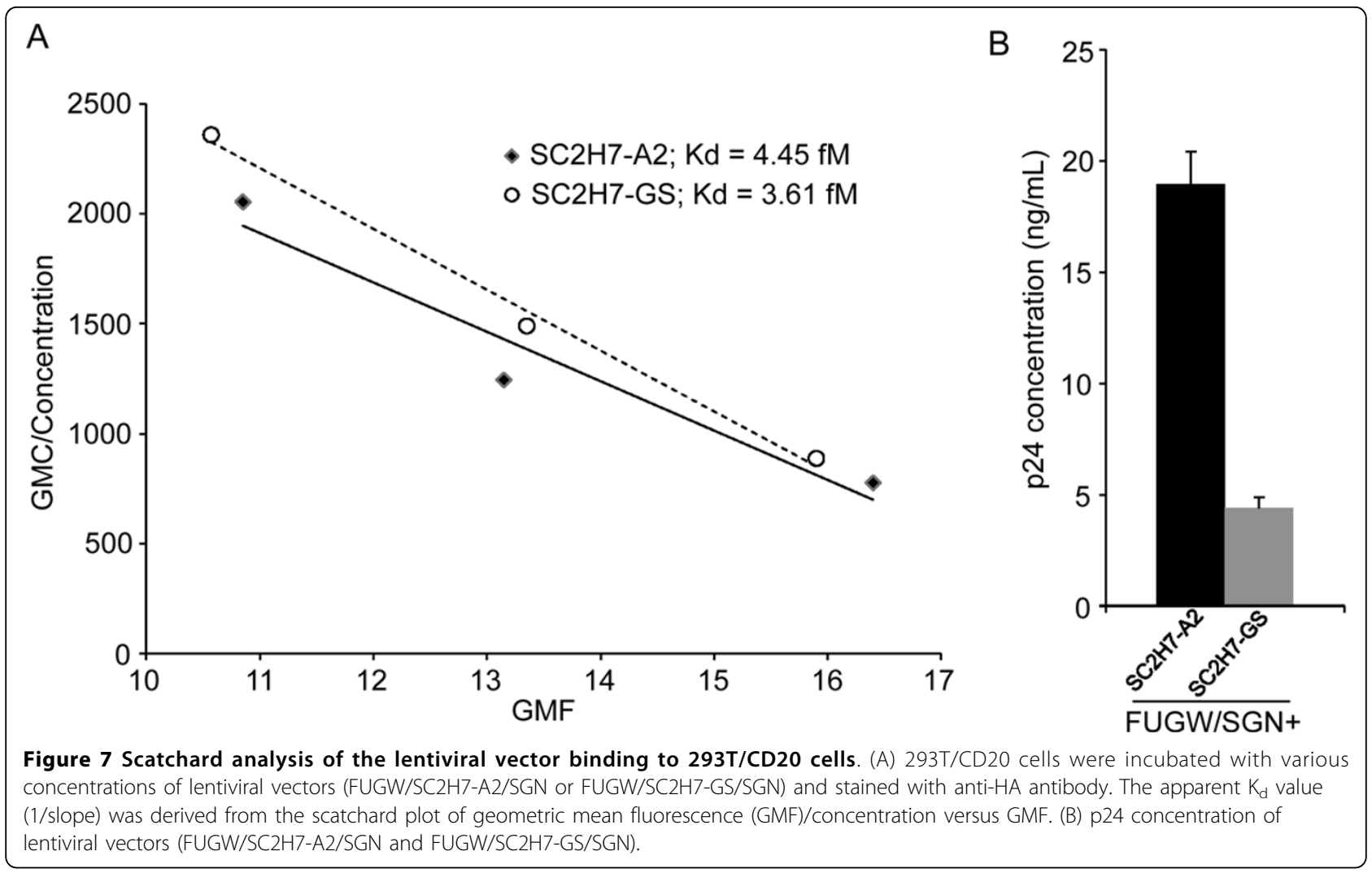

product was assembled with the light chain variable region using the primers HLA-A2fw and CD20hvbw. To fuse the hinge- $\mathrm{CH} 2-\mathrm{CH} 3$ domain to the HLA-A2 transmembrane domain, we PCR-amplified the hinge- $\mathrm{CH} 2-$ $\mathrm{CH} 3$ domain and the HLA-A2 transmembrane domain using the primer pairs (CH2-CH3-Hingefw, 5'-GTC TCT TCA GTT GAG CCC AAA TCT TGT GAC AAA ACT CAC ACA TGC CCA CCG TGC CCA GCA CCT GAA CTC CTG GGG GGA CCG TC-3'; CH2-CH3bw, 5'-CTG GGA AGA CGG GGC CCC CTG TCC GAT CAT GTT CCT G-3') and (HLA-A2Tfw, 5'-GAC AGG GGG CCC CGT CTT CCC AGC CCA CCA TCC CC3'; HLA-A2Tbw, 5'-CGA GCG GCC GCT CAC ACT TTA CAA GCT GTG AGA GAC ACA TCA GAG CCC-3'). The resulting two PCR fragments were assembled using primers $\mathrm{CH} 2-\mathrm{CH} 3-\mathrm{Hingefw}$ and HLAA2Tbw. We then assembled the variable fragments with the $\mathrm{CH} 2-\mathrm{CH} 3 /$ transmembrane domain using the primers HLA-A2fw and HLA-A2Tbw. The assembled DNA was finally cloned into pcDNA3 (Invitrogen) via Hind 3 and Not1 restriction sites. To construct a single chain antibody with the VSVG transmembrane domain, a forward primer (SC2H7fw, 5'-CCC CCA TCC CGG GAT GAG CTG ACC-3') and a backward primer (SC2H7bw, 5'-AGT ATC ACC GGC CCC CTG TCC GAT CAT GTT CCT GTA GTC-3') were used to amplify a portion of the $\mathrm{CH} 2-\mathrm{CH} 3$ domain of $\mathrm{SC} 2 \mathrm{H} 7$ -
A2. In parallel, a forward primer (GSfw, 5'-ATG ATC GGA CAG GGG GCC GGT GAT ACT GGG CTA TCC AAA AAT CCA ATC GAG CTT-3') and a backward primer (GSbw, 5'-GAT CGA GCG GCC GCT TAC TTT CCA AGT CGG TTC ATC TCT ATG TCT GTA TAA ATC TGT CTT TTC-3') were used to amplify the transmembrane domain of VSVG. The DNA products from these two reactions were PCR-assembled using $\mathrm{SC} 2 \mathrm{H} 7 \mathrm{fw}$ and GSbw as the primer pair and the resulting product was cloned into $\mathrm{pSC} 2 \mathrm{H} 7-\mathrm{A} 2$ to yield SC2H7-GS. The integrity of these constructs was confirmed by DNA sequencing.

\section{Viral vector production}

$293 \mathrm{~T}$ cells were seeded in a $6-\mathrm{cm}$ culture dish in DMEM medium supplemented with fetal bovine serum (Sigma, St. Louis, MO, 10\%), L-glutamine $(10 \mathrm{~mL} / \mathrm{L})$, penicillin, and streptomycin (100 units $/ \mathrm{mL})$ the night prior to transfection. 293T cells were transfected at a confluence of $80 \sim 90 \%$ with $5 \mu \mathrm{g}$ of lentiviral backbone vector (FUGW), $2.5 \mu \mathrm{g}$ each of $\mathrm{pMDLg} / \mathrm{pRRE}$, pRSVRev, pFM, and a plasmid encoding an antibody (pSC2H7-A2, pSC2H7-GS or pAB) via the standard calcium phosphate precipitation technique [36]. Cells were replenished with pre-warmed media 4 hours post-transfection. Vectors were harvested two days post-transfection and filtered through a $0.45-\mu \mathrm{m}$ pore size filter 
(Nalgene, Rochester, NY). Lentiviral vectors were then further concentrated by ultracentrifugation (Optimal L90K Ultracentrifuge, Beckman Coulter, Fullerton, CA) at $4^{\circ} \mathrm{C}, 25,000 \mathrm{rpm}$ for 90 minutes and resuspended in appropriate volume of cold PBS.

\section{Virus-cell binding assay}

293T/CD20 or 293T cells were incubated with $2 \mathrm{~mL}$ of lentiviral vectors (FUGW/SC2H7-A2/FM, FUGW/ $\mathrm{SC} 2 \mathrm{H} 7-\mathrm{GS} / \mathrm{FM}$ or FUGW/AB/FM) at $4^{\circ} \mathrm{C}$ for 1 hour. After extensive washing with cold PBS, cell-virus complexes were stained with anti-HA tag antibody (Miltenyi Biotec, Inc.) and analyzed by flow cytometry (FACSort, BD Bioscience).

\section{Confocal imaging}

Fluorescent images were acquired on a Zeiss LSM 510 META laser scanning confocal microscope equipped with Argon, red $\mathrm{HeNe}$, and green $\mathrm{HeNe}$ lasers as well as a Coherent Chameleon Ti-Sapphire laser for multiphoton imaging. Images were acquired using a Plan-apochromat $63 \mathrm{x} / 1.4$ oil immersion objective. To image viruscell binding, cells were seeded into a $35-\mathrm{mm}$ glass-bottom culture dish and grown at $37^{\circ} \mathrm{C}$ overnight. The seeded cells were rinsed with cold PBS and incubated with concentrated viral particles for 1 hour at $4^{\circ} \mathrm{C}$ to allow for binding. The cells were washed with cold PBS to remove unbound particles, fixed with $4 \%$ formaldehyde on ice for 10 minutes, and then immunostained with monoclonal antibody specific for HIV capsid protein p24 and 4',6-diamidino-2-phenylindole (DAPI) antibody for nuclear staining. Monoclonal antibody against HIV-1 p24 (AG3.0) was obtained from the NIH AIDS Research and Reference Reagent Program (Division of AIDS, NIAID, NIH). Images were analyzed using the Zeiss LSM 510 software version 3.2 SP2.

\section{Antibody Competition Assay}

293T/CD20 cells were incubated with the lentiviral vector (FGUW/SC2H7-A2/SGN or FUGW/VSVG) and various amount of either the soluble $\alpha$ CD20 (BD Bioscience) or the isotype control antibody overnight. Cells were then replenished with fresh media and incubated for additional 72 hours before flow cytometry analysis.

\section{Neutralization Assay}

293T/CD20 or Ghost-CCR5 (NIH AIDS Research and Reference Reagent Program) cells were pre-incubated with various amount of bafilomycin for 30 minutes, after which, the lentiviral vector (FUGW/SC2H7-A2/ SGN or FUGW/GP160) was added. The vector and cell mixture was spun at $25^{\circ} \mathrm{C}, 2,500 \mathrm{rpm}$ for 90 minutes using a RT legend centrifuge (Sorval). Cells were then incubated at $37^{\circ} \mathrm{C}$ and $5 \% \mathrm{CO}_{2}$ and replenished with fresh media 3 hours later. Flow cytometry was then used to analyze the treated cells 3 days posttransduction.

\section{Targeted transduction of $293 \mathrm{~T} / \mathrm{CD} 20$ cells}

$293 \mathrm{~T}$ or $293 \mathrm{~T} / \mathrm{CD} 20$ cells were seeded on a 24 -well cell culture plate and spin-transduced with $1.5 \mathrm{~mL}$ of indicated lentiviral vectors (FUGW/SC2H7-A2/FM, FUGW/ SC2H7-GS/FM, FUGW/AB/FM, FUGW/SC2H7-A2, or $\mathrm{FUGW} / \mathrm{SC} 2 \mathrm{H} 7-\mathrm{GS})$ at $25^{\circ} \mathrm{C}, 2,500 \mathrm{rpm}$ for 90 minutes using a RT legend centrifuge. After replacing with fresh media, the treated cells were cultured for additional 5 days at $37^{\circ} \mathrm{C}$ and $5 \% \mathrm{CO}_{2}$. Flow cytometry was then used to analyze transduction efficiency. The titer was determined by measuring GFP-positive cells in the dilution range that resulted in a linear relationship between the percentage of GFP-expressing cells and the amount of vectors added.

\section{Scatchard analysis}

293T/CD20 cells were incubated with various amount of lentiviral vectors (FUGW/SC2H7-A2/SGN or FUGW/ SC2H7-GS/SGN). Flow cytometry analysis was carried out to measure the geometric mean fluorescence (GMF) of the bound viruses stained by anti-HA antibody. The concentration of the lentiviral vectors was measured by a p24 antigen capture enzyme immunosorbent assay (ELISA) kit (ImmunoDiagnostics, Woburn, MA). Apparent $K_{d}$ value was derived from the negative reciprocal of the slope of the linear fit to scatchard plots, which is the geometric mean fluorescence/concentration of lentiviral vector (GMF/concentration) against geometric mean fluorescence (GMF).

\section{Virus-liposome fusion assay}

1,2-dioleoyl-sn-glycero-3-phosphocholine (DOPC) and 1,2-dioleoyl-sn-glycero-3-phosphoethanolamine (DOPE) were purchased from Avanti Polar Lipids (Alabaster, AL, USA). Cholesterol (Chol) and sphingomyelin (SPM) from egg yolk were obtained from Sigma (St Louis, MO, USA). Liposomes were prepared by the extrusion procedure [37]. Briefly, lipid mixtures (PC/PE/SPM/Chol molar ratio of 1:1:1:2) were dried from a chloroform solution under a stream of argon gas and further dried under vacuum for at least 3 hours. The lipid mixtures were hydrated in HNE buffer (5 mM HEPES, $150 \mathrm{mM}$ $\mathrm{NaCl}$, and $0.1 \mathrm{mM}$ EDTA, pH 7.4). Subsequently, the lipid mixtures were extruded 20 times through $0.2 \mu \mathrm{m}$ pore size polycarbonate filters (Avanti polar lipids). To monitor virus-liposome fusion, the concentrated viruses were incubated with $70 \mu \mathrm{M}$ of octadecyl rhodamine B chloride (R18) (Molecular Probes, Carlsbad, CA, USA) in serum-free medium for 1 hour at room temperature. 
R18-labeled viruses were then mixed with liposomes (200 $\mu \mathrm{M}$ phospholipids) in a final volume of $0.4 \mathrm{~mL}$. Fusion was triggered by adding the appropriate volume of $0.2 \mathrm{M}$ acetic acid, pretitrated to achieve the desired $\mathrm{pH}$. The dequenching signal of R18 fluorescence was measured 60 seconds after acidification with QuantaMaster QM-4SE spectrofluorometer (Photon Technology International, Lawrenceville, NJ, USA). The initial fluorescence of virus-liposome mixtures was set at $0 \%$ fusion, and the $100 \%$ fusion value was obtained by detergent lysis for each experiment using $0.1 \%$ of Triton $\mathrm{X}-100$ [38].

\section{Authors' contributions}

$\mathrm{YL}$ and $\mathrm{PW}$ designed research; $\mathrm{YL}, \mathrm{KJ}, \mathrm{JZ}$ and $\mathrm{CW}$ performed research; $\mathrm{YL}$ and PW analyzed data and wrote the paper. All authors read and approved the final manuscript.

\section{Competing interests}

The authors declare that they have no competing interests.

Received: 16 December 2009

Accepted: 11 February 2010 Published: 11 February 2010

\section{References}

1. Verma I, Somia. N: Gene therapy - promises, problems and prospects. Nature 1997, 389:239-242.

2. Verma I, Weitzman M: Gene therapy: twenty-first century medicine. Annu Rev Biochem 2005, 74:711-738.

3. Burns J, Friedmann T, Driever W, Burrascano M, Yee J: Vesicular stomatitis virus $\mathrm{G}$ glycoprotein pseudotyped retroviral vectors: concentration to very high titer and efficient gene transfer into mammalian and nonmammalian cells. Proc Nat Acad Sci USA 1993, 90:8033-8037.

4. Cronin J, Zhang $X$, Reiser J: Altering the tropism of lentiviral vectors through pseudotyping. Curr Gene Ther 2005, 5:387-398.

5. Mastromarino P, Conti C, Goldoni P, Hauttecoeur B, Orsi N: Characterization of membrane components of the erythrocyte involved in vesicular stomatitis virus attachment and fusion at acidic pH. J Gen Virol 1987, 68:2359-2369

6. Hall M, Burson $\mathrm{K}$, Huestis W: Interactions of a vesicular stomatitis virus $G$ protein fragment with phosphatidylserine: NMR and fluorescence studies. Biochim Biophys Acta 1998, 1415:101-113.

7. Nguyen $T$, Pages $J C$, Farge $D$, Briand $P$, Weber A: Amphotropic retroviral vectors displaying hepatocyte growth factor-envelope fusion proteins improve transduction eficiency of primary hepatocytes. Hum Gene Ther 1998, 17:2469-2474.

8. Somia N, Zoppe M, Verma I: Generation of targeted retroviral vectors by using single-chain variable fragment: an approach to in vivo gene delivery. Proc Nat Acad Sci USA 1995, 92:7570-7574.

9. Jiang A, Dornburg R: In vivo cell type-specific gene delivery with retroviral vectors that display single chain antibodies. Gene Ther 1999, 6:1982-1987

10. Martin F, Chowdhury S, Neil S, Phillipps N, Collins MK: Envelope-targeted retrovirus vectors transduce melanoma xenografts but not sleen or liver. Mol Ther 2002, 5:269-274.

11. Valsesia-Wittmann S, Drynda A, Deleage G, Aumailley M, Heard J, Danos O, Verdier G, Cosset F: Modifications in the binding domain of avian retrovirus envelope protein to redirect the host range of retroviral vectors. J Virol 1994, 68:4609-4619.

12. Kasahara N, Dozy A, Kan Y: Tissu-specific targeting of retroviral vectors through ligand-receptor interactions. Science 1994, 266:1373-1376.

13. Jiang A, Chu T, Nocken F, Cichutek K, Dornburg. R: Cell type-specific gene transfer into human cells with retroviral vectors that display single chain antibodies. J Virol 1998, 72:10148-10156.

14. Maurice M, Verhoeyen E, Salmon P, Trono D, Russell SJ, Cosset FL: Efficient gene transfer into human primary blood lymphocytes by surface- engineered lentiviral vectors that display a T cell-activating polypeptide. Blood 2002, 99:2342-2350.

15. Fielding AK, Fernandes SC, Chadwick MP, Bullough FJ, Cosset FL: A Hyperfusogenic Gibbon Ape Leukemia Envelope Glycoprotein: Targeting of a Cytotoxic Gene by Ligand Display. Hum Gene Ther 2000, 11:817-826.

16. Gollan TJ, Green MR: Selective targeting and inducible destruction of human cancer cells by retroviruses with envelope proteins bearing short peptide ligands. J Virol 2002, 76:3564-3569.

17. Morizono K, Bristol G, Xie Y, Kung S, Chen I: Antibody-directed targeting of retroviral vectors via cell surface antigens. J Virol 2001, 75:8016-8020.

18. Morizono $K$, Xie $Y$, Ringpis $G$, Johnson M, Nassanian $H$, Lee B, Wu L, Chen ISY: Lentiviral vector retargeting to P-glycoprotein on metastatic melanoma through intravenous injection. Nat Med 2005, 11:346-352.

19. Roux $P$, Jeanteur P, Piechaczyk M: A versatile and potentially general approach to the targeting of specific cell types by retroviruses: application to the infection of human cells by means of major histocompatibility complex class I and class II antigens by mouse esctropic murine leukemia virus-derived viruses. Proc Nat Acad Sci USA 1989, 86:9079-9083.

20. Boerger A, Snitkovsky S, Young J: Retroviral vectors preloaded with a viral receptor-ligand bridge protein are targeted to specific cell types. Proc Nat Acad Sci USA 1999, 96:9867-9872.

21. Yang $L$, Bailey $L$, Baltimore $D$, Wang P: Targeting lentiviral vectors to specific cell types in vivo. Proc Nat Acad Sci USA 2006, 103:11479-11484.

22. Lu L, Cassese T, Kielian M: The Chloesterol Requirement for Sindbis Virus Entry and Exit and Characterization of a Spike Protein Region Involved in Cholesterol Dependence. J Virol 1999, 73:4272-4278.

23. Lei $Y$, Joo Kl, Wang P: Engineering fusogenic molecules to achieve targeted transduction of enveloped lentiviral vectors. J Biol Eng 2009, 3:8.

24. Yang H, Zeigler L, Joo K-I, Cho T, Lei Y, Wang P: Gamma-retroviral vectors enveloped with an antibody and an engineered fusogenic protein achieved antigen-specific targeting. Biotechnol Bioeng 2008, 101:357-368.

25. Liao K, Chou W, Lo Y, Roffler S: Design of transgenes for efficient expression of active chimeric proteins on mammalian cells. Biotechnol Bioeng 2000, 73:313-323.

26. Chou W, Liao K, Lo Y, Jiang S, Yeh M, Roffler S: Expression of chimeric monomer and dimer proteins on the plasma membrane of mammalian cells. Biotechnol Bioeng 1999, 65:160-169.

27. Liao K, Chen B, Liu T, Tzou S, Lin Y, Lin K, Su C, Roffler S: Stable expression of chimeric anti-CD3 receptors on mammalian cells for stimulation of antitumor immunity. Cancer Gene Ther 2003, 10(10):779-90.

28. Roffler S, Wang H, Yu H, Chang W, Cheng C, Lu Y, Chen B, Cheng T: A membrane antibody receptor for noninvasive imaging of gene expression. Gene Ther 2006, 13:412-420.

29. Fredericksen B, Wei B, Yao J, Luo T, Garcia J: Inhibition of endosomal/ lysosomal degradation increases the infectivity of human immunodeficiency virus. J Virol 2002, 76:11440-11446.

30. McClure $M$, Marsh $M$, Weiss $R$ : Human immunodeficiency virus infection of CD4-bearing cells occurs by a $\mathrm{pH}$-independent mechanism. EMBO J 1988, 7:513-518.

31. Gibbons DL, Vaney MC, Roussel A, Vigouroux A, Reilly B, Lepault J, Kielan M, Rey FA: Conformational change and protein-protein interactions of the fusion protein of Semliki Forest virus. Nature 2004, 427:320-325.

32. Kielian M, Rey FA: virus memrane-fusion proteins: more than one way to make a hairpin. Nat Rev Microbiol 2006, 4:67-76.

33. Liu CY, Kielian M: E1 mutants identify a critical region in the trimer interface of the semliki forest virus fusion protein. J Virol 2009, 83(21):11298-11306.

34. Leung K, Kim JO, Ganesh L, Kabat J, Schwartz O, Nabel GJ: HIV-1 assembly: viral glycoproteins segregate quantally to lipid rafts that associate individually with virions. Cell Host Microbe 2008, 3:285-292.

35. Jorgenson RL, Vogt VM, Johnson MC: Foreign glycoproteins can be actively recruited to virus assembly sites during pseudotyping. J Virol 2009, 83:4060-4067.

36. Klages $N$, Zufferey $R$, Trono D: A stable system for the high-titer production of multiply attenuated lentiviral vectors. Mol Ther 2000, 2:170-176.

37. Smit JM, Bittman R, Wilschut J: Low-pH-dependent fusion of sindvis virus with receptor-free cholesterol- and sphingolipid-containing liposomes. J Virol 1999, 73:8476-8484 
38. Wunderli-Allenspach $\mathrm{H}$, Ott $\mathrm{S}$ : Kinetics of fusion and lipid transfer between virus receptor containing liposomes and influenza viruses as measured with the octadecylrhodamine B chloride assay. Biochemistry 1990, 29:1990-1997.

doi:10.1186/1743-422X-7-35

Cite this article as: Lei et al: Targeting lentiviral vector to specific cell types through surface displayed single chain antibody and fusogenic molecule. Virology Journal 2010 7:35.

Submit your next manuscript to BioMed Central and take full advantage of:

- Convenient online submission

- Thorough peer review

- No space constraints or color figure charges

- Immediate publication on acceptance

- Inclusion in PubMed, CAS, Scopus and Google Scholar

- Research which is freely available for redistribution

Submit your manuscript at www.biomedcentral.com/submit
C Biomed Central 